\title{
Editorial corner - a personal view Need for a unified theory of the electrical properties of composites with fractal structure
}

\author{
Gy. Bánhegyi*
}

Medicontur Medical Engineering Ltd., Herceghalmi Road, 2072 Zsámbék, Hungary

Composite materials have long been used for electrical insulation purposes - from oil impregnated paper insulated cables to nano-ceramic filled epoxy resin insulator bodies. Theories describing the basic electrical properties of layered composites go back to Maxwell, while good approximations were developed for dilute matrix-inclusion type composites in the first half of the $20^{\text {th }}$ century. When extending these models to concentrated systems it turned out that statistical mixture type composites exhibit basically different properties (including percolation) from matrix/inclusion composites. Conductive fillers as carbon black and graphite have long been used for rendering insulating matrices to semiconducting or conducting composites. Assessment of percolation was based on DC resistivity tests. Since then huge steps were taken. Percolation theory, fractal mathematics and physics developed a lot from the 1980's. We have a full range of carbon, metal semiconductor nanostructures, occasionally impregnated with ionic fluids, conjugated polymers, polyelectrolytes etc. Our mathematical and modeling capabilities at the micro-, meso- and macro-level have also developed tremendously allowing the conscious design and tailoring of various meta-materials, photonic crystals, fractal structures for electrical sensing, and so on. It seems to me that here is the time to unite these pieces of knowledge and to build up a unified theory, which would cover the morphology of fractal nanostructures by filling continuous media with various shapes of nanofillers, by mixing irregular particles

\footnotetext{
${ }^{*}$ Corresponding author, e-mail: gybanheg@t-online.hu (C) BME-PT
}

of widely differing properties, by chemical reactions and impregnation of nanoporous media. This theory should describe not only the DC and AC electrical properties, breakdown characteristics, nonlinearities, aging phenomena for any combination of insulating, electrolyte and metallic components. Such a framework would be useful not only in electrical engineering, but also in supercapacitors, Li-electrode materials, sensor applications, corrosion, ceramic based composites and meta-material development. Experimental verification of such models would allow to use broadband dielectric spectroscopy or impedance spectroscopy as a diagnostic tool in a wide range of technical or even biomedical problems. Fitting various parameters in the model to the experimentally observed impedance data may help in determining inclusion shape and orientation, connectivity of the dispersed shape, surface conductivity, charge carrier concentration and mobility which cannot be always determined by other techniques or direct observation. A good model may also help in designing.

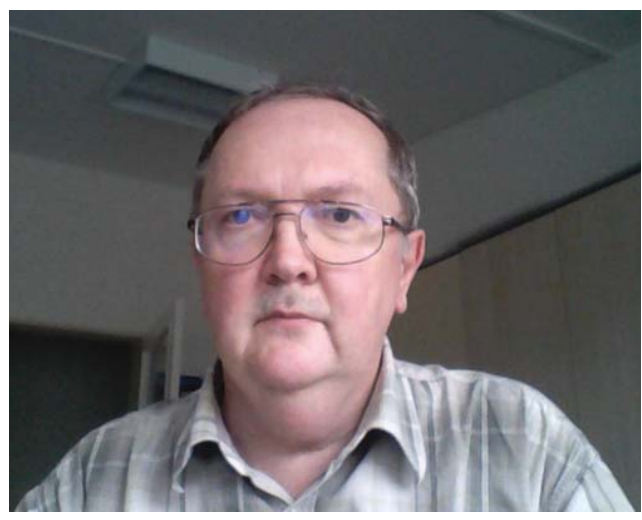

Dr. György Bánhegyi

Member of Executive Editorial Board 